\section{G113(P) INNOVATIVE QUALITY IMPROVEMENT PROJECT TO IMPROVE ORAL HEALTH KNOWLEDGE AND PRACTICES OF CHILDREN WITH DIABETES}

${ }^{1,2} \mathrm{~K}$ Woon Sam, ${ }^{1,2} \mathrm{~S}$ Uppal, ${ }^{2} \mathrm{C}$ Utting, ${ }^{1} \mathrm{~N}$ Aswani, ${ }^{3} \mathrm{~A}$ Dickenson, ${ }^{1} \mathrm{O}$ Okike ${ }^{1} \mathrm{Derhyshire}$ Children's Hospital, University Hospitals of Derby and Burton NHS FT, Derby, UK; ${ }^{2}$ Dental Services, Derbyshire Community Health Services NHS FT, Derby, UK; ${ }^{3}$ Postgraduate School of Dentistry, Health Education England, Leicester, UK

\subsection{6/archdischild-2020-rcpch.90}

Aims Patients with diabetes are at a higher risk of developing a number of oral health $(\mathrm{OH})$ conditions, most notably periodontal disease. This project sets out to assess and increase patient knowledge about $\mathrm{OH}$ in a paediatric diabetic outpatient clinic.

Methods Using a Plan-Do-Study-Act (PDSA) methodology, children being seen for diabetic outpatient review were given the opportunity to see a dentist in addition to their regular team. Families who accepted this offer were given oral health education, toothbrushing instruction and advice about how to tackle risks specific to them. For each patient seen between September and October 2019, the dentist completed an oral health recording tool (OHRT). This provided the opportunity to study the patient's current practices and knowledge about diabetes and $\mathrm{OH}$. In future reviews, the OHRT will be repeated to assess improvement in patient's knowledge following our intervention. This is a Trust approved quality improvement project.

Results The results of the completed pre-intervention OHRTs are presented here. Out of the 28 children seen, $89 \%$ visit a dentist regularly. However, most of the families (61\%) had no knowledge that diabetes had any impact on oral health and just $7 \%$ were aware of the specific risks to gingival health. $57 \%$ were unaware of risk reducing strategies and $14 \%$ were brushing their teeth less than once a day.

Conclusion This project aligns with the Making Every Contact Count initiative and is the first of its kind to our knowledge. The majority of families from our initial cohort lacked awareness about the risks of diabetes to $\mathrm{OH}$ and about different preventative strategies, justifying the need for further education in this high risk patient group. We have demonstrated that giving this preventive advice is possible without the need for additional appointments for children with diabetes. In addition, undergraduate dental students are now part of the $\mathrm{OH}$ education team thereby increasing the number of patients that can be seen on any one clinic and expanding the students' clinical experience.

\section{G114(P) REDUCING FASTING TIMES IN PAEDIATRIC SURGICAL PATIENTS: 'THE TICKING CLOCK'}

K Sun, N Yoganayagam, J Pallawela. Paediatrics, North Middlesex University Hospital, London, UK

\subsection{6/archdischild-2020-rcpch.91}

Aims To reduce the time paediatric surgical patients spent fasted unnecessarily through a quality improvement initiative that provided healthcare staff with a tool to review fasting times regularly.

Methods After nursing staff frequently highlighted concerns that patients were fasted longer than necessary at a district general hospital, often without regular review of this decision, a new proforma entitled 'The Ticking Clock' was introduced.
It provided nurses with a tool, to re-evaluate and re-assess a patient's fasting status every two hours with the surgical team. Introduction of the 'Ticking Clock' was the primary intervention, however highlighting the issue at hand to both the paediatrics and surgical departments, as well as engaging the Practice Development Nurse to help with educating nurses about the new intervention were also undertaken to help implement the change. Data was collected over a two week period prior to our intervention, with data collection being repeated six months later. Our primary outcome was the average time paediatric surgical patients spent fasted in total, with a focus on emergency surgical patients.

Results The preliminary study collected data for 14 patients in total. These were predominantly admitted under General Surgery but also included those admitted under Orthopaedics, Urology and Gynaecology. The re-auditing process collected data for a total of ten patients, six months after the intervention was implemented. On comparing the two data sets, there was a notable reduction in time patients spent fasted. On average post implementation, patients spent 7 hours 13 minutes in total fasting compared to 14 hours 48 minutes prior to introduction of 'The Ticking Clock'.

Conclusion The implementation of 'The Ticking Clock' significantly reduced the average time paediatric surgical patients spent fasted by $50 \%$ over the course of 6 months in a district general hospital. Further studies to investigate its improvement on patient experience directly can be built from this.

\section{G115(P) A DISCHARGE SUMMARY ON DISCHARGE}

D Toma, H Abdalla. Paediatrics, Manor Hospital, Walsall Healthcare NHS Trust, Walsall, UK

\subsection{6/archdischild-2020-rcpch.92}

Children attending the paediatrics assessment unit (PAU) for less than 4 hours were not receiving a copy of their discharge summary (DS) before going home. This meant that most of the time information was passed verbally to the patient and their family, with no written support to prevent misunderstanding and consolidate information. There were also significant delays in getting important information sent to GP surgeries. Our project's aim was to provide a copy of the DS for all patients with a $<4 \mathrm{~h}$ stay. This was more ambitious than the original organisational target, but more in keeping with patients' needs and those of GPs.

One of the contributing factors was the complicated process of completing a DS: there were two paper forms, both handwritten, one for the doctors and one for the nurses, each taking 3-5 minutes to complete and containing duplicated information. Another inconvenience was that DS' were often completed by doctors who had not seen the patient.

We proceeded to facilitate the process by devising a new form which unified the nurses' and doctors' discharge form into a single page paper form. This was refined over several PDSA cycles to reflect feedback from all stakeholders. It was eventually replaced by an identical PDF digital form. Adjustment to PAU equipment had to be done.

Baseline data collection showed that $0 \%$ of patients were going home with a DS on discharge. In the first two weeks after implementing the unified form we have achieved levels of $60-100 \%$ and afterwards levels of $96-100 \%$ have been constantly maintained. Self-reported completion times reduced from 6-10 minutes to 3-6 minutes. 